\title{
JAK-Inhibitoren für die Behandlung hämatoonkologischer Erkrankungen
}

\section{JAK Inhibitors for the Treatment of Haemato-Oncological Diseases}

Autoren

Torsten Steinbrunn, Josip Zovko, Sabrina Kraus

Institut

Medizinische Klinik und Poliklinik II, Universitätsklinikum

Würzburg, Würzburg

Schlüsselwörter

JAK-Inhibitor, Myeloproliferative Neoplasien, Primäre Myelofibrose, Polycythaemia vera, Graft-versus-Host-Erkrankung

\section{Keywords}

JAK inhibitor, primary myelofibrosis, graft-versus-host disease, polycythaemia vera, myeloproliferative neoplasias

online publiziert $\quad 04.11 .2020$

Bibliografie

Akt Rheumatol 2020; 45: 559-567

DOI 10.1055/a-1285-4125

ISSN 0341-051X

(c) 2020. Thieme. All rights reserved.

Georg Thieme Verlag KG, Rüdigerstraße 14,

70469 Stuttgart, Germany

Korrespondenzadresse

Dr. Torsten Steinbrunn

Medizinische Klinik und Poliklinik II

Universitätsklinikum Würzburg

Oberdürrbacher Straße 6

97080 Würzburg

Deutschland

Tel.: 0931-201-40141, Fax: 0931-201-640141

steinbrunn_t@ukw.de

\section{ZUSAMMENFASSUNG}

Die konstitutive Aktivierung des JAK-STAT-Signalwegs ist charakteristisch für die Pathogenese der myeloproliferativen Neoplasien, speziell der primären Myelofibrose, der Polycythaemia vera und der essentiellen Thrombozythämie. Die Einführung von oral verfügbaren JAK-Inhibitoren in die Klinik brachte einen entscheidenden Fortschritt für die pharmakologische Behandlung der Myelofibrose und der Polycythaemia vera, wenngleich damit noch keine Heilung verbunden ist. Im Vordergrund steht die Verbesserung der Lebensqualität der meist älteren Patienten durch Kontrolle krankheitsbedingter konstitutioneller Symptome, Reduktion einer bestehenden Splenomegalie und Vermeidung insbesondere von thromboembolischen Folgekomplikationen. Darüber hinaus kann die Therapie von Myelofibrose-Patienten mit JAK-Inhibitoren jedoch auch deren Krankheitsverlauf verlangsamen und ihr Gesamtüberleben verlängern. Der bislang einzige in Europa zugelassene JAK-Inhibitor Ruxolitinib hemmt die Isoformen JAK1 und JAK2 und besitzt sowohl antiinflammatorisches als auch antiproliferatives Potenzial. Damit zeigt dieser Inhibitor überdies eine gute Wirkung in der Therapie der Graft-versus-Host-Erkrankung nach allogener hämatopoetischer Stammzelltransplantation. MitFedratinib, Pacritinib und Momelatinib befinden sich derzeit 3 weitere vielversprechende JAK-Inhibitoren mit etwas unterschiedlichen Wirkprofilen in der klinischen Phase III-Testung. Diese zeigen auch bei Patienten mit unwirksamer oder unverträglicher Vorbehandlung mit Ruxolitinib Wirksamkeit, sodass eine kontinuierliche Weiterentwicklung der entsprechenden Therapiestrategien abzusehen ist.

\section{ABSTRACT}

The constitutive activation of the JAK-STAT signalling pathway is a pathogenetic hallmark of myeloproliferative neoplasms, in particular of primary myelofibrosis, polycythaemia vera and essential thrombocythaemia. The introduction of orally available JAK inhibitors into clinics yielded a major progress for the pharmacological treatment of myelofibrosis and polycythaemia vera, although they have not been able to cure these conditions. The primary goal is to improve the quality of life in the predominantly elderly patients by controlling the constitutional symptoms caused by the disease, to reduce existing splenomegaly and to prevent subsequent thromboembolic complications. Moreover, the therapeutic administration of JAK inhibitors to patients with myelofibrosis may decelerate the course of their disease and prolong their overall survival. So far, Ruxolitinib is the only JAK inhibitor approved in Europe. It targets both isoforms JAK1 and JAK2 and has antiinflammatory as well as antiproliferative potential. Consequently, this inhibitor also proves effective in treating graft-versus-host disease occurring after allogeneic haematopoietic stem cell transplantation. Next in line, Fedratinib, Pacritinib and Momelatinib are three promising JAK inhibitors with slightly different activity profiles, which are currently undergoing clinical phase III testing. They prove effective even in patients with prior ineffective or intolerable exposure to Ruxolitinib. Thus, a continuous improvement of the respective treatment strategies can be expected. 


\section{Einleitung}

Der deregulierte und aktivierte Janus kinase (JAK)-signal transducer and activator of transcription (STAT)-Signalweg spielt nicht nur eine Schlüsselrolle in der Pathogenese von Autoimmunerkrankungen wie rheumatoider Arthritis (RA), systemischem Lupus erythematodes, chronisch-entzündlicher Darmerkrankungen und Vaskulitiden [1], sondern ist auch ein wesentlicher Treiber für die myeloproliferativen Neoplasien (MPN), speziell der Polycythaemia vera (PV), der essentiellen Thrombozythämie (ET) und der primären sowie sekundären (post-PV- und post-ET-) Myelofibrose (MF) [2]. Zudem wird die inflammatorische Aktivität der Graft-versus-HostErkrankung (GvHD) nach allogener hämatopoetischer Stammzelltransplantation (allo-HSCT) zu einem überwiegenden Teil über den JAK-STAT-Signalweg vermittelt [3]. Durch pharmakologische Hemmung der JAK-Proteine lässt sich die Aktivität dieses Signalwegs herabregulieren, sodass sowohl die genannten entzündlich-rheumatischen Erkrankungen als auch die hämatoonkologischen Entitäten wirksam therapiert werden können. Für die rheumatologische Indikation RA sind in Europa die JAK-Inhibitoren Baricitinib, Upadacitinib und Tofacitinib zugelassen, letzteres zudem bei Colitis ulcerosa und Psoriasis-Arthritis [4]. Die Zulassung für Filgotinib wird darüber hinaus in Kürze erwartet.

In den hämatoonkologischen Indikationen MF und PV besteht eine Zulassung bislang nur für Ruxolitinib [5]. Hier wird die Zulassung für Fedratinib erwartet. Zudem wird für Ruxolitinib eine Zulassung in der Indikation der steroidrefraktären akuten GvHD (aGvHD) erwartet.

Die folgende Übersicht soll eine Momentaufnahme über den Einsatz von JAK-Inhibitoren in der Hämatoonkologie wiedergeben.

\section{Myeloproliferative Neoplasien}

Die MPN umfassen eine Gruppe seltener maligner hämatologischer Stammzellerkrankungen, denen eine überschießende klonale Proliferation einer oder mehrerer Blutzellreihen gemeinsam ist. Die jährliche Inzidenz beträgt insgesamt etwa 1-2/100 000. Männer sind etwas häufiger betroffen als Frauen. In der klinischen Verwendung bezieht sich der Terminus MPN üblicherweise auf die Hauptentitäten ET, PV und primäre MF (PMF) einschließlich der Vorstufe prä-PMF [6]. Die Klassifikation der Weltgesundheitsorganisation (WHO) zählt darüber hinaus auch die chronische myeloische Leukämie (CML) sowie die sehr seltenen Formen der chronischen Neutrophilen- und Eosinophilenleukämie dazu [7]. Die Pathogenese der CML konnte bereits in den 1960er Jahren zunächst auf das Philadelphia-Chromosom (22q-) und später mittels spezifischerer Methoden auf die Genfusion BCR-ABL1 (breakpoint cluster region, Abelson tyrosine kinase 1) auf dem Boden der Translokation t(9;22) zurückgeführt werden. In der Folge wurden PV, ET und PMF in Abgrenzung zur CML als Philadelphia-Chromosom-negative beziehungsweise BCR-ABL-negative MPN zusammengefasst. Die den intrazellulären JAK-STAT-Signalweg aktivierenden molekularen Aberrationen dieser MPN wurden erst erheblich später in den 2000er Jahren identifiziert [2].

Definitionsgemäß bestehen bei der PV erhöhte Hämatokrit- beziehungsweise Hämoglobinwerte ( $>49 \%$ beziehungsweise $>16,5$ $\mathrm{g} / \mathrm{dl}$ für Männer und $>48 \%$ beziehungsweise $>16 \mathrm{~g} / \mathrm{dl}$ für Frauen), wobei meist gleichzeitig auch die Granulo- und Megakaryopoese gesteigert ist.
Bei der ET ist hingegen isoliert die Thrombozytenzahl erhöht

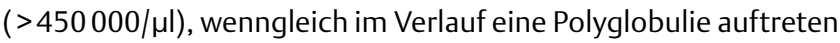
und den Übergang in eine PV andeuten kann.

Auch die (frühe) PMF äußert sich initial meist als Thrombozytose und ist im Anfangsstadium - der sogenannten prä-PMF - klinisch kaum von der ET zu unterscheiden. Im weiteren Verlauf entwickelt sich jedoch eine zunehmende Knochenmarkfibrosierung mit Einlagerung von Retikulin- und Kollagenfasern, sodass durch die Verdrängung der Hämatopoese eine zunehmende Panzytopenie entsteht. Auch die PV und weniger häufig die ET können nach jahrelangem Krankheitsverlauf in eine sekundäre Myelofibrose (MF) übergehen, die sogenannte Post-PV-MF beziehungsweise Post-ET-MF.

Patienten mit MPN werden meist zufällig im Rahmen von Routineuntersuchungen diagnostiziert, da diese Erkrankungen zumindest initial asymptomatisch verlaufen und nur zögerlich voranschreiten. Die PV und die PMF treten meist jenseits des 60. Lebensjahres auf, wohingegen Patienten mit ET mit 40-50 Jahren eher etwas jünger sind. Erste Beschwerden sind in der Regel unspezifisch und äußern sich als Leistungsminderung, Kopfschmerzen oder manchmal auch Zeichen einer B-Symptomatik mit Nachtschweiß und Gewichtsverlust. In fortgeschritteneren Stadien mit stärker erhöhter Zellzahl treten Zeichen unterschiedlich ausgeprägter Durchblutungsstörungen in den Vordergrund, die von Schwindel, Tinnitus und Sehstörungen über Dysästhesien der Finger und Zehen bis hin zu schwerwiegenden arteriellen und venösen thromboembolischen Komplikationen - insbesondere auch an atypischen Lokalisationen (z. B. Pfortader- oder Sinusvenenthrombose, Budd-Chiari-Syndrom und andere) - reichen können. Die Mehrzahl der Patienten mit PV berichtet zudem von einem charakteristischen und nur schwer zu kontrollierenden aquagenen Pruritus.

Im längeren Verlauf und bei fortschreitender Knochenmarkfibrosierung übernimmt die Milz einen Teil der eingeschränkten Hämatopoese. Eine dadurch entstehende Splenomegalie zeigt sich bei ca. einem Drittel der Patienten mit PV und etwas häufiger sowie ausgeprägter bei der PMF und den sekundären MF-Formen [8]. Dadurch entstehende Folgebeschwerden und Komplikationen können sowohl die Lebensqualität als auch die Prognose deutlich beeinträchtigen. Die mediane Überlebenszeit der MF beträgt 6 Jahre, wobei die Spannweite individuell sehr unterschiedlich ist [9]. Im weiteren Erkrankungsverlauf können die MPN in eine akute myeloische Leukämie übergehen, bevorzugt auf dem Boden einer PMF, post-PV-MF oder post-ET-MF, in welchen bis zu $20 \%$ der Patienten betroffen sind [10]. Hier ist Prognose mit einer mittleren Überlebenszeit von 2,6 bis 7 Monaten [11] und 2-Jahres-Überlebensrate von $15 \%$ [12] nur sehr begrenzt.

\section{Der aktivierte JAK-STAT-Signalweg}

Die den MPN gemeinsam zugrundeliegende molekulare Veränderung betrifft die konstitutive Aktivierung des intrazellulären JAKSTAT-Signalwegs. Diese Signalkaskade leitet extrazelluläre Wachstums- und Zellregulationssignale, die über Typ I- oder Typ II-Zytokinrezeptoren nach intrazellulär vermittelt werden, zum Zellkern weiter und reguliert damit die Gentranskription. Mehr als 50 verschiedene Zytokine nutzen diesen Signalweg in unterschiedlichen Zelltypen des Immunsystems, dazu zählen insbesondere Zytokine mit proinflammatorischer Wirkung wie bspw. Interleukin (IL)-2, IL-6, IL-12 oder Interferon (IFN)-y, aber auch einige Zytokine, die 
antiinflammatorische Wirkung vermitteln können, wie z. B. IL-4, IL-10 oder IL-13 [13]. Zunächst bewirkt die Zytokinbindung an die extrazelluläre Domäne ihrer jeweiligen Transmembranrezeptoren deren Dimerisierung, worauf intrazelluläre JAK-Proteine, die bereits mit der zytoplasmatischen Rezeptordomäne verbunden sind, ATP-abhängig phosphoryliert werden. Diese Phosphatgruppen werden daraufhin auf intrazelluläre Tyrosinreste der dimerisierten Rezeptoren selbst übertragen. Anschließend können sich dort STAT-Proteine anlagern, die ihrerseits ebenfalls dimerisieren und als Transkriptionsfaktoren in den Zellkern translozieren. Beim Menschen sind an diesem Mechanismus die 4 JAK-Proteine JAK1, JAK2, JAK3 und TYK2 (tyrosine kinase 2) sowie 7 verschiedene STAT-Proteine beteiligt [13]. Die charakteristische Treibermutation der MPN betrifft JAK2 und führt zu einer von der Zytokinwirkung entkoppelten konstitutiven Aktivierung des JAK-STAT-Signalwegs in den betroffenen myeloischen Zellen.

JAK2-Genmutationen lassen sich in so gut wie allen Patienten mit PV nachweisen [14]. Bei einem kleinen Teil (5\%) besteht die JAK2-Mutation exklusiv in Exon 12 und äußert sich in der Aktivierung des Erythropoetinrezeptors (EPOR) [15]. Die meisten Patienten $(95 \%)$ zeigen allerdings die „klassische“ JAK2-Mutation in Exon 14 mit dem daraus resultierenden V617F-Transkript. Zusätzlich zur Aktivierung des EPOR führt diese Mutation auch zur Aktivierung des granulocyte colony-stimulating growth factors (G-CSF) und des Thrombopoetinrezeptors (MPL, myeloproliferative leukemia virus oncogene). Dies erklärt, warum die JAK2-V617F Mutation daher auch für die ET und PMF charakteristisch ist und sich hier in jeweils etwa $60 \%$ der Patienten findet [16].

Weitere $23-30 \%$ der Patienten mit ET oder PMF besitzen Mutationen im Calreticulin (CALR)-Gen (Exon 9), die ebenfalls zu einer aktivierenden Wirkung auf den JAK-STAT-Signalweg führen, oder Mutationen im MPL-Gen (resultierender Aminosäureaustausch meist W515L/K), wenngleich der diesbezügliche genaue Aktivierungsmechanismus des Signalwegs bei den MPN nicht vollständig verstanden ist [17]. Ca. 10\% der Patienten mit PMF oder ET besitzen keine dieser „klassischen“ Mutationen und werden als „triple-negative“ (dreifach negativ) eingeordnet, was mit einer ungünstigeren Prognose verbunden ist [18]. Mithilfe der Next-generation Sequencing (NGS)-Technologie können allerdings auch bei dieser Patientengruppe in einigen Fällen atypische JAK2- oder MPL-Mutationen an unterschiedlichen Genlokalisationen nachgewiesen werden [19].

Darüber hinaus können andere zusätzlich auftretende, subklonale Läsionen in weiteren Genen den Krankheitsverlauf ungünstig beeinflussen. Dazu zählen unter anderem Mutationen in ASXL1, DNMT3A, EZH2, IDH1/2, SF3B1 und TP53, die sich bspw. auf Transkriptionsfaktoren oder epigenetische Regulatoren auswirken [6]. Für das Risiko einer leukämischen Transformation aus einer PV oder MF scheint in Verbindung mit einer JAK2-Mutation insbesondere der Verlust von TP53 bedeutsam zu sein [15].

In diesem Zusammenhang sei erwähnt, dass JAK2-Mutationen zwar ein Charakteristikum für die MPN darstellen, dass jedoch der aktivierte JAK-STAT-Signalweg auch in lymphatischen Erkrankungen eine treibende Rolle übernehmen kann. So bestehen JAK2-Mutationen bei einem Teil ( $9 \%$ ) der Patienten mit akuter lymphatischer Leukämie (ALL), zudem finden sich JAK2-Amplifikationen in über einem Drittel der Patienten mit Hodgkin-Lymphom und primär mediastinalem B-Zell-Lymphom [20].

\section{Therapie der primären und sekundären Myelofibrose}

Die medikamentöse Therapie sowohl der Myelofibrose wie auch der PV und ET ist nicht kurativ intendiert, sondern zielt auf die Symptomkontrolle und die Verbesserung der Lebensqualität [21]. Neben der Vermeidung von Komplikationen wie Thromboembolien oder Blutungen soll zudem das Voranschreiten der PMF und die Entwicklung einer Post-PV- beziehungsweise Post-ET-MF verzögert werden. Die Indikation zur medikamentösen Therapie der MF hängt daher in erster Linie von der Risikoeinteilung und der vorhandenen Symptomatik ab.

Die prognostische Klassifikation der MF erfolgt zum Diagnosezeitpunkt nach dem International Prognostic Scoring System (IPSS) [22] in die 4 Kategorien Niedrig-, Intermediär 1-, Intermediär 2und Hochrisiko. Hierbei werden die Parameter Alter, Leukozytenzahl, Hämoglobinwert, Blastenanteil im peripheren Blut und konstitutionelle Symptome einbezogen. Zusätzlich haben sich das Dynamic International Prognostic Scoring System (DIPSS) [23] sowie der DIPSS-plus-Score [24] etabliert, die beide auch im weiteren Erkrankungsverlauf bestimmt werden können. Der DIPSS-plus-Score erfasst zusätzlich zu den genannten Parametern die Thrombozytenzahl, den Transfusionsbedarf für Erythrozyten und bestimmte Aberrationen des Karyotyps. Im Zuge der zunehmenden molekularen Kenntnisse wurden zudem mutations- beziehungsweise genetisch basierte IPSS-Scores wie z. B. MIPSS70 [25], MIPSS70-plus [26] oder GIPSS [27] entwickelt.

Eine medikamentöse Therapie der MF ist gemäß der ELN-Empfehlungen bei Intermediär 2- oder Hochrisiko-Konstellation oder bei krankheitsbedingter konstitutioneller Symptomatik indiziert [28]. Aufgrund der Alters- und Komorbiditätsstruktur kommen für die Mehrheit der betroffenen Patienten nur palliativ intendierte Therapiestrategien infrage [10]. Neben der zytoreduktiven Substanz Hydroxyurea (bei Hyperproliferation) steht hierfür seit 2012 der JAK1/2-Inhibitor Ruxolitinib zur Verfügung, der auf Basis der beiden unten näher beschriebenen COMFORT-Studien zugelassen wurde.

Auch zur Therapie der PV ist Ruxolitinib zugelassen, sofern der Einsatz von Hydroxyurea zur Zytoreduktion nicht ausreicht oder nicht vertragen wird. Grundlage hierfür bildeten die Daten der Phase III-Studie RESPONSE, in der die Behandlung von PV-Patienten mit Ruxolitinib verglichen mit der bestverfügbaren Therapie eine signifikante Symptomverbesserung und Reduktion des Hämatokritwertes erreichte [29].

Für eine detaillierte Darstellung weiterer Therapiestrategien sei auch auf das Leitlinienportal Onkopedia der Deutschen Gesellschaft für Hämatologie und Medizinische Onkologie (DGHO) [30] verwiesen.

Die einzige kurative Option stellt die allo-HSCT dar, die jedoch aufgrund erhöhter Morbidität und Mortalität in der älteren Patientengruppe nur für eine Minderheit geeignet ist, wenngleich die Anzahl an durchgeführten allo-HSCTs mit der Indikation MF insgesamt steigen [31]. Die meisten Patienten erhalten altersentsprechende Konditionierungsprotokolle mit reduzierter Therapieintensität (RIC) [32]. Gemäß der Konsensusempfehlungen der European Bone Marrow Transplantation (EBMT)/European Leukemia Network (ELN) Arbeitsgruppe besteht die Indikation zur allo-HSCT für Patienten mit Intermediär 2- oder Hochrisiko-Erkrankung, die jünger als 70 Jahre sind. Ebenso sollten Patienten < 65 Jahren mit Intermediär 1-Risiko transplantiert werden, sofern sie einen hohen Transfu- 
-Tab. 1 Übersicht über die in dieser Arbeit beschriebenen pharmakologischen JAK-Inhibitoren mit einer Auswahl an molekularen und klinischen Eigenschaften.

\begin{tabular}{|c|c|c|c|c|}
\hline JAK-Inhibitor & Isoformspezifität & Relevante Off-Targets & Bemerkungen & Zulassung \\
\hline Ruxolitinib & JAK1 JAK2 & ROCK & $\begin{array}{l}\text { Hämatotoxizität (Anämie, Thrombozytopenie) hauptsäch- } \\
\text { lich durch JAK2-Hemmung bedingt [41]; Immunmodulati- } \\
\text { on zum Teil über ROCK-Hemmung in dendritischen Zellen } \\
\text { [42]; Derzeit einziger in der EU zugelassener JAK-Inhibitor } \\
\text { für hämatoonkologische Erkrankungen }\end{array}$ & $\begin{array}{l}\text { EMA und FDA: PV, PMF, } \\
\text { post-PV-MF, post-ET-MF } \\
\text { Nur FDA: steroidrefraktäre } \\
\text { aGvHD }\end{array}$ \\
\hline Fedratinib & JAK2 & FLT3 RET & $\begin{array}{l}\text { Gastrointestinale Symptome größtenteils durch } \\
\text { FLT3-Hemmung bedingt [53]; Auftreten von Wernicke- } \\
\text { Enzephalopathie im Zusammenhang mit Thiaminmangel } \\
\text { sehr selten möglich [54] }\end{array}$ & $\begin{array}{l}\text { Nur FDA: PMF, post-PV-MF, } \\
\text { post-ET-MF }\end{array}$ \\
\hline Pacritinib & JAK2 & FLT3 IRAK1 CSF1R & $\begin{array}{l}\text { Geringere Rate an Hämatotoxizität beobachtet, daher } \\
\text { möglicherweise besonders auch für Patienten mit } \\
\text { Thrombozytopenie geeignet [56] }\end{array}$ & keine \\
\hline Momelotinib & JAK1 JAK2 & ACVR1 & $\begin{array}{l}\text { Mittels ACVR1-Hemmung indirekte Stimulation der } \\
\text { Erythropoese, dadurch möglicherweise Verringerung der } \\
\text { Transfusionshäufigkeit bei Patienten mit Anämie [58] }\end{array}$ & keine \\
\hline Itacitinib & JAK1 & nicht bekannt & $\begin{array}{l}\text { In klinischer Testung u.a. zur Behandlung von aGvHD und } \\
\text { cGvHD }\end{array}$ & keine \\
\hline Ilginatinib & JAK2 & SRC FYN & In klinischer Testung zur Behandlung von MF & keine \\
\hline Gandotinib & JAK2 & FLT3 & In klinischer Testung zur Behandlung von MF, PV und ET & keine \\
\hline \multicolumn{5}{|c|}{$\begin{array}{l}\text { ACVR1 - activin A receptor type 1; aGvHD - akute Graft-versus-Host-Erkrankung; cGvHD - chronische Graft-versus-Host-Erkrankung; CSF1R - colony } \\
\text { stimulating factor } 1 \text { receptor; EMA - European Medicines Agency (Europäische Arzneimittel-Agentur); ET - Essentielle Thrombozythämie; FDA - U.S. Food } \\
\text { and Drug Administration; FLT3 - FMS-like tyrosine kinase 3; FYN - Tyrosinkinase Fyn; IRAK1 - interleukin-1 receptor-associated kinase 1; JAK - Januskinase; } \\
\text { JAK1 - Janus kinase 1; JAK2 - Janus kinase 2; MF - Myelofibrose; PMF - primäre Myelofibrose; PV - Polycythaemia vera; RET - Rezeptortyrosinkinase Ret; } \\
\text { ROCK - Rho-associated coiled-coil containing protein kinase; SRC - Tyrosinkinase Src. }\end{array}$} \\
\hline
\end{tabular}

sionsbedarf für Erythrozyten, Blasten im peripheren Blut ( $>2 \%$ ) oder eine ungünstige Zytogenetik aufweisen [9].

Heutzutage sind die meisten Patienten, die sich mit der Indikation zur allo-HSCT vorstellen, bereits mit dem JAK1/2-Inhibitor Ruxolitinib vorbehandelt [32]. Der vorherige Einsatz von Ruxolitinib scheint sich hierbei allerdings nicht wesentlich auf die post-Transplantationsergebnisse auszuwirken [33], wenngleich die sich unmittelbar stellenden Fragen nach dem potenziellen Einfluss auf die Rezidivwahrscheinlichkeit oder das Auftreten von GvHD noch nicht abschließend beantwortet sind [32].

\section{JAK-Inhibition für die Therapie der Myelofibrose und der Polycythaemia vera}

Für das Verständnis der Funktion von JAK-Inhibitoren ist bedeutsam, dass Patienten mit MPN selbst dann eine erhöhte Aktivierung des JAK-STAT-Signalwegs aufweisen, wenn keine JAK2-Mutation vorliegt [2]. Entsprechend hängt die klinische Wirksamkeit und die Indikationsstellung für pharmakologische JAK-Inhibitoren bei MF-Patienten nicht vom Nachweis einer JAK2-Mutation ab [34]. Der zielgerichtete Effekt der JAK-Inhibition ist somit nicht als mutationsspezifisch, sondern vielmehr als signalwegsspezifisch anzusehen ( $>$ Tab. 1).

Als einziger JAK-Inhibitor ist in Europa derzeit der oral verfügbare Wirkstoff Ruxolitinib für die Therapie der primären beziehungsweise sekundären MF bei relevanter Splenomegalie oder anderer MF-bedingter Symptomatik sowie für die PV nach Vorbehand- lung mit Hydroxyurea zugelassen. Ruxolitinib hemmt die Isoformen JAK1 sowie JAK2 und führt dadurch zur Abnahme der Zellproliferation. Auf diese Weise erreichen die weitaus meisten MF-Patienten eine Reduktion ihrer Milzgröße [21], wobei dies in einer dosisabhängigen Wirkbeziehung steht [35]. In den beiden randomisierten Phase III-Studien COMFORT-I [36] und COMFORT-II [37] wurde der Einsatz von Ruxolitinib mit Placebo beziehungsweise mit der bestverfügbaren Therapie verglichen. Die dort erhobenen Daten wurden in der vor kurzem veröffentlichten Phase-III-Studie JUMP im Wesentlichen bestätigt [38]. Die Behandlung mit dem JAK1/2-Inhibitor zeigte dabei jeweils eine signifikante Reduktion der Symptomlast und eine Abnahme der Milzgröße um durchschnittlich ca. ein Drittel nach 24 Wochen [36]. In längeren Nachbeobachtungen dieser Studien wird für die Untergruppe der Patienten mit Intermediär-2- und Hochrisiko-MF auch ein verlängertes Gesamtüberleben beschrieben [34, 39]. Bei nicht wenigen Patienten zeigten sich relevante Effekte erst im Verlauf von mehreren Monaten, woraus geschlossen wurde, dass ein Nichtansprechen frühestens einen Monat nach Therapiestart festgestellt werden kann und eine definitive Beurteilung des Therapieerfolgs erst nach 6 Monaten empfohlen wird [37]. Die Dosierung wird in Abhängigkeit von der Thrombozytenzahl determiniert und beträgt zwischen 10 und $40 \mathrm{mg}$ pro Tag verteilt auf 2 Gaben. Trotz hoher initialer Ansprechraten hatte in den genannten Studien etwa die Hälfte der Patienten Ruxolitinib nach 3 Jahren entweder wegen nachlassender Wirksamkeit oder intolerabler Nebenwirkungen beendet [8]. Zu letzteren gehören in ers- 
ter Linie Einschränkungen der Hämatopoese mit Anämie und Thrombozytopenie in $60 \%$ beziehungsweise $45 \%$ der Fälle. Die Lymphopoese soll durch Ruxolitinib aufgrund der fehlenden Wirkung auf JAK3 jedoch kaum beeinträchtigt werden [40]. Allerdings gehören verschiedene Infektionen wie Pneumonien, Harnwegsinfekte und Varizella-zoster-Reaktivierung durchaus zum Nebenwirkungsprofil. Bisweilen treten darüber hinaus intolerable gastrointestinale Nebenwirkungen wie Übelkeit, Erbrechen und Diarrhoe auf oder auch asymptomatische Anstiege der Lebertransaminasen und der Lipase in der Laborchemie. Während die myelosuppressiven Nebenwirkungen eine direkte Folge der JAK2-Hemmung darstellen [41], werden die gastrointestinalen Symptome häufig durch unspezifische sogenannte off-target-Effekte vermittelt. Darüber hinaus gibt es allerdings auch recht spezifische off-target-Effekte, die für einen wichtigen Teil der klinischen Wirksamkeit der JAK-Inhibitortherapie verantwortlich sein könnten. Bspw. hemmt Ruxolitinib auch die Rho-associated coiled-coil containing protein kinase (ROCK) in dendritischen Zellen und könnte somit durch deren Funktionseinschränkung einen Teil der immunmodulatorischen Wirkung vermitteln [42]. Zu einem möglicherweise erhöhten Risiko für die Entwicklung aggressiver B-Zelllymphome unter einer JAKInhibitortherapie bestehen widersprüchliche Ergebnisse [43, 44]. Deren Auftreten scheint insbesondere mit einer bereits vorbestehenden monoklonalen B-Lymphozytose zu korrelieren, sodass vor der Gabe eines JAK-Inhibitors eine durchflusszytometrische Untersuchung zur Detektion eines solchen B-Zellklons zu befürworten ist [45]. Eine weitere seltene Nebenwirkung ist das sogenannte ruxolitinib discontinuation syndrome (RDS), das sich nach dem abrupten Absetzen von Ruxolitinib aufgrund der dann wiederaufflammenden Zytokinerhöhung entwickeln kann [46]. Diese Reaktion ähnelt einem septischen Krankheitsbild und tritt in unterschiedlichen Schweregraden meist innerhalb von einer bis 3 Wochen auf. Daher empfiehlt es sich, JAK-Inhibitoren bei Beendigung langsam auszuschleichen [47]. Unabhängig hiervon kehren nach Beendigung oder Versagen der Therapie mit Ruxolitinib die MF-bedingten Symptome rasch auf das Ausgangsniveau zurück [36] und die Prognose von MF-Patienten sinkt ohne weitere Therapie auf ein medianes Gesamtüberleben von 13 bis 14 Monaten [48, 49].

Mit den in Europa noch nicht zugelassenen JAK-Inhibitoren der zweiten Linie Fedratinib, Pacritinib und Momelotinib stehen (in Zukunft) Substanzen zur Verfügung, die auch nach Vorbehandlung mit Ruxolitinib Wirksamkeit zeigen. Fedratinib ist ein gegen JAK2 und FMS-like tyrosine kinase (FLT)-3 gerichteter oral verfügbarer Inhibitor, der seit August 2019 in den USA für Patienten mit Intermediär-2- oder Hochrisiko-MF auf der Basis von Daten der vorzeitig beendeten Phase III-Studie JAKARTA [50] sowie der Phase II-Studie JAKARTA-2 [51] zugelassen ist. In diesen Studien wurde Fedratinib in JAK-Inhibitor-naiven beziehungsweise in mit Ruxolitinib vorbehandelten Patienten getestet und zeigte in Bezug auf Symptomkontrolle und Reduktion der Milzgröße Ergebnisse, die mit denen von Ruxolitinib aus den klinischen Studien COMFORT-I und COMFORT-II vergleichbar waren [21, 52]. Häufigste Nebenwirkungen, die Dosisreduktionen erforderten, bestanden wiederum in Anämie und Thrombozytopenie, die als sogenannte on-targetEffekte für die JAK2-Hemmung typisch sind [41] sowie in gastrointestinalen Symptomen, die am ehesten als off-target-Effekte aus der FLT3-Hemmung resultieren [53]. Wegen des Verdachts auf ein erhöhtes Auftreten von neurologischen Komplikationen, im Speziellen einer Wernicke-Enzephalopathie, wurden die klinischen Studien zu Fedratinib zwischenzeitlich ausgesetzt. Diese Symptome dürften letztlich auf nutritiv bedingten Thiaminmangel verstärkt durch die gastrointestinalen Nebenwirkungen zurückzuführen sein [54], entsprechend wird in der klinischen Anwendung empfohlen, besonderes Augenmerk auf neurologische Ausfallserscheinungen und die Ernährungssituation der Patienten zu legen [52]. 2 klinische Phase-III-Studien, FREEDOM und FREEDOM-2, evaluieren derzeit das Ansprechen auf Fedratinib bei MF-Patienten mit Ruxolitinib-Vorbehandlung [55].

Auch Pacritinib hemmt wie Fedratinib JAK2 und FLT3. Zusätzlich besitzt Pacritinib eine zielgerichtete Wirkung gegen interleukin-1 receptor-associated kinase (IRAK)- 1 , jedoch keine Wirkung gegen JAK1, weshalb die unerwünschten Effekte auf die Megakaryopoese geringfügiger ausgeprägt sind $[55,56]$, sodass sich diese bislang nicht zugelassene Substanz möglicherweise insbesondere für Patienten mit vorbestehender Thrombozytopenie eignet [21]. Die Phase-III-Studie PERSIST2 evaluierte die Effektivität von Pacritinib in MF-Patienten mit Ruxolitinib-Vorbehandlung, wobei eine relevante Verringerung der Milzgröße in $10 \%$ der Patienten erreicht wurde [57].

Momelotinib ist ein Inhibitor von JAK1 und JAK2 und besitzt indirekt über die zusätzliche Hemmung von activin A receptor type1 (ACVR1) Erythropoese-stimulierende Eigenschaften [58]. In der Phase-III-Studie SIMPLIFY-1 zeigte Momelotinib eine Verbesserung des Transfusionsbedarfs gegenüber Ruxolitinib und war gemäß dem Studienendpunkt in Bezug auf die Reduktion der Milzgröße gegenüber Ruxolitinib nicht unterlegen [8,59]. Daher eignet sich diese Substanz möglicherweise besonders für Patienten mit vorbestehender Anämie [21,60].

Zu den weiteren neuen JAK-Inhibitoren in klinischer hämatoonkologischer Testung zählen der JAK1-Inhibitor Itacitinib sowie die beiden JAK2-Inhibitoren Ilginatinib und Gandotinib [21]. Zudem sind Ansätze zur Kombinationsbehandlung von JAK-Inhibitoren mit weiteren Substanzen von hohem Interesse, um eine gesteigerte therapeutische Wirksamkeit über mehrere Angriffspunkte auf die Erkrankung zu erzielen. Hierbei ist jedoch durchaus auch mit unerwarteten Toxizitäten zu rechnen, weshalb unter anderem eine Studie zur Kombination von Ruxolitinib mit dem Immunmodulator Lenalidomid vorzeitig beendet wurde [61]. Hingegen befindet sich der Immunmodulator Pomalidomid weiterhin in Kombination mit Ruxolitinib in klinischer Testung [30]. Auch zahlreiche weitere Substanzen werden in aktuellen Studien mit Ruxolitinib kombiniert, darunter z. B. JAK-Inhibitoren mit unterschiedlicher Isoformspezifität, hypomethylierende Agenzien, Histondeacetylase (HDAC)-Inhibitoren sowie kleine Moleküle gegen B-cell lymphoma (BCL)-2, Phosphoinositid-3-Kinase (PI3K), Cyclin-abhängige Kinasen (CDK)$4 / 6$ und andere [21].

\section{JAK-Inhibition zur Therapie der akuten Graft-versus- Host-Erkrankung nach allogener hämatopoetischer Stammzelltransplantation}

Die allo-HSCT ist bei vielen Patienten mit malignen hämatologischen Erkrankungen wie Leukämien und Lymphomen oft die einzige kurative Option, insbesondere nach Versagen konventioneller Therapien. Neben Infektionen und Krankheitsrezidiven ist die in 
der Regel innerhalb der ersten 100 Tage nach allo-HSCT auftretende akute GvHD (aGvHD) die häufigste schwerwiegende transplantationsassoziierte Komplikation. Je nach Kriterien entwickeln zwischen 20 und $80 \%$ aller Patienten infolge der allo-HSCT trotz adäquater medikamentöser Immunsuppression eine aGvHD [62], neuere Einschätzungen beziffern diese Rate auf etwa $50 \%$ [63]. Betroffen ist in den meisten Fällen die Haut mit einer sonnenbrandähnlichen makulopapulösen Rötung der Haut, in schweren Fällen bis zur Blasenbildung und Exkoriation. Weitere häufig betroffene Organsysteme sind der Gastrointestinaltrakt mit frequenten wässrigen Diarrhoen, teils mit Blutbeimengungen, Abdomenschmerzen, Übelkeit, Erbrechen sowie Inappetenz und die Leber, deren Beteiligung sich in einer Transaminasenerhöhung und Bilirubinämie, z. T. verbunden mit rechtsseitigen Oberbauchschmerzen oder Ikterus, äußert [63].

Pathophysiologisch beginnt die aGvHD damit, dass immunkompetente Donor-T-Zellen des allogenen Transplantats (graft) mit antigenpräsentierenden Zellen interagieren und hierüber das Körpergewebe des Patienten (host) als „fremd“ erkennen. In der Folge werden sowohl das native als auch das adaptive (Spender-)Immunsystem aktiviert, wobei neben den genannten T-Zellen auch neutrophile Granulozyten und dendritische Zellen eine wichtige Rolle übernehmen [63]. Im Ergebnis wird ein Entzündungsprozess eingeleitet, der zu schwersten Gewebeschäden führen kann.

Auf die Standardtherapie mit Kortikosteroiden spricht etwa die Hälfte der Patienten mit aGvHD (Grad II-IV) nachhaltig an, wohingegen die andere Hälfte als steroidrefraktär anzusehen ist [64]. Mehr als ein Drittel der Patienten mit schwerer aGvHD (Grad III/IV) verstirbt innerhalb der ersten 100 Tage nach allo-HSCT [65]. Die Zweitlinientherapie unterlag über lange Zeit keinen einheitlichen Kriterien und beinhaltete verschiedene immunsuppressive und antiinflammatorische Strategien, z. B. mit Calcineurininhibitoren, mammalian target of rapamycin (mTOR)-Inhibitoren, tumor necrosis factor (TNF)- $\alpha$-Hemmern, extrakorporaler Photopherese $(E C P)$ und anderen. Eine wesentliche Weiterentwicklung war die Erkenntnis, dass ein relevanter Teil der komplexen Immunreaktion über die Aktivierung des JAK-STAT-Signalwegs in den T-Effektorzellen und verschiedenen weiteren Immunzellen verläuft, der somit eine rationale Zielstruktur für JAK-Inhibitoren zur Behandlung der aGvHD darstellt [66]. In der Phase-II-Studie REACH1 sprachen 57\% der Patienten mit steroidrefraktärer aGvHD auf die Therapie mit dem JAK1/2-Inhibitor Ruxolitinib in einer Tagesdosis von $10 \mathrm{mg}$ bis $20 \mathrm{mg}$ in Kombination mit Kortikosteroiden an, das mediane Gesamtüberleben betrug knapp 11 Monate [67]. Basierend auf diesen Daten ist Ruxolitinib zur Behandlung der steroidrefraktären aGvHD in den USA seit 2019 zugelassen [68]. Eine entsprechende Zulassung in Europa besteht noch nicht, wird allerdings erwartet.

In der Phase-III-Studie REACH2 wurde die Behandlung der steroidrefraktären aGvHD mit der bestverfügbaren Therapie verglichen [69]. Ruxolitinib führte zu signifikant höheren Ansprechraten sowohl kurzfristig (62,3 vs. 39,4\% an Tag 28) als auch langfristig (39,6 vs. 21,9\% an Tag 56). Zudem bestand ein nicht-signifikanter Trend zu einem höheren Gesamtüberleben in der Ruxolitinib-Gruppe $[69,70]$.

Bezüglich der chronischen GvHD (cGvHD), die in der Regel protrahiert mehrere Monate nach allo-HSCT auftritt, zeigte eine retrospektive Analyse eine Ansprechrate von 43,4\% nach 12-monatiger
Therapie mit Ruxolitinib bei Steroidrefraktärität [71]. In der aktuell laufenden Phase-III-Studie REACH3 wird derzeit die Wirksamkeit von Ruxolitinib auf die steroidrefraktäre chronische GvHD prospektiv getestet [72].

In diesem Zusammenhang sei erwähnt, dass sich der Nutzen von Ruxolitinib nicht auf die steroidrefraktäre Situation beschränkt, sondern auch in den weitaus meisten steroidsensitiven Patienten eine zügige und oft vollständige Reduktion der Kortikosteroiddosis und der damit verbundenen kurz- und langfristigen Nebenwirkungen ermöglicht wird [73].

Wenngleich Ruxolitinib im Allgemeinen gut vertragen wird, steigt insbesondere bei der längerfristigen Anwendung das Risiko für Infektkomplikationen durch Viren, bspw. Cytomegalie- und Herpes-simplex-Viren, Bakterien wie z. B. Enterokokken und Pilze wie bspw. Aspergillen [74]. Daher wird während der Anwendung der JAK-Inhibitortherapie eine kontinuierliche antivirale, antimykotische und gegebenenfalls auch antibiotische Prophylaxe empfohlen [74].

Aufgrund der funktionellen Komplexität der Entstehung und Aufrechterhaltung einer GvHD über verschiedene immunologische beziehungsweise inflammatorische Mechanismen liegen Strategien zur Kombinationsblockade mit Kortikosteroiden oder weiteren immunsuppressiven Substanzen nahe. So zeigte bspw. die bereits beschriebene REACH1-Studie Vorteile für die Kombination von Ruxolitinib mit Kortikosteroiden [67]. Auch der neue JAK1-Inhibitor Itacitinib wird in Kombination mit Kortikosteroiden in Patienten mit aGvHD und cGvHD getestet [75].

\section{Ausblick}

JAK-Inhibitoren haben die therapeutischen Möglichkeiten in der Behandlung der MPN grundlegend erweitert. Seither besteht für diese Patienten erstmals eine kausal basierte und wirksame Therapieoption zur Reduktion der Symptomlast bis hin zur Verbesserung der Prognose. Hiervon profitiert vor allem die große Gruppe der älteren Patienten mit MF, die sich nicht für die einzige kurative Therapieoption - die allo-HSCT - eignen. Laufende klinische Studien lassen eine Vielzahl neuer JAK-Inhibitoren mit teils unterschiedlicher Isoformspezifität für den Einsatz als Alternative zum bislang einzigen zugelassenen JAK-Inhibitor Ruxolitinib oder in der nächsten Therapielinie nach dessen Versagen oder bei Unverträglichkeit erwarten. Für Patienten, die eine steroidrefraktäre aGvHD nach allo-HSCT entwickeln, bedeutet die in den USA bereits erfolgte und für Europa bald erwartete Zulassung von Ruxolitinib ebenfalls eine grundlegende Veränderung der klinischen Praxis mit deutlicher Verbesserung des Krankheitsansprechens. Von hohem Interesse sind künftige Kombinationstherapien von JAK-Inhibitoren mit Kortikosteroiden und anderen Immunsuppressiva, um die komplexen GvHD-Mechanismen über mehrere Zielstrukturen zu hemmen und die meist hämatotoxischen Nebenwirkungen nach Möglichkeit zu reduzieren. Darüber hinaus kann spekuliert werden, dass JAKInhibitoren nicht nur in der Therapie der GvHD, sondern künftig bereits für deren Prävention ein relevante Rolle einnehmen werden. 
FAZIT

- Der JAK1/2-Inhibitor Ruxolitinib ist für die Therapie der PMF sowie der post-PV/ET-MF indiziert, sofern konstitutionelle Symptome oder ein erhöhtes prognostisches Risiko ( $\geq$ Intermediär-2) vorliegen.

- Bei der Polycythaemia vera ist Ruxolitinib in der Zweitlinientherapie nach Vorbehandlung mit Hydroxyurea zugelassen.

- Die Therapie der MPN mit JAK-Inhibitoren ist palliativ. In erster Linie vermindert sie konstitutionelle Symptome, reduziert eine Splenomegalie und verbessert die Lebensqualität.

- Fedratinib, Pacritinib und Momelantinib sind neue JAK-Inhibitoren mit unterschiedlichen Wirkprofilen, die sich in klinischer Phase-III-Testung befinden und vielversprechende Wirkung auch bei Patienten nach unwirksamer oder unverträglicher Ruxolitinib-Vorbehandlung zeigen.

- JAK-Inhibitoren besitzen eine gute klinische Wirksamkeit auch bei der Therapie der steroidrefraktären GvHD nach allo-HSCT, wenngleich hierfür in Europa noch keine Zulassung besteht.

\section{Interessenkonflikt}

T. Steinbrunn: Finanzielle Interessen: Referentenhonorar oder Kostenerstattung als passiver Teilnehmer: Vortragshonorare: Janssen, Medac; Kongressteilnahmen: Gilead, Janssen, Jazz, Novartis, Servier; Nichtfinanzielle Interessen: Oberarzt, Universitätsklinikum Würzburg, Medizinische Klinik und Poliklinik II; Mitgliedschaften: Deutsche Gesellschaft für Hämatologie und Medizinische Onkologie (DGHO), International Myeloma Society (IMS).

J. Zovko: Finanzielle Interessen: Referentenhonorar oder Kostenerstattung als passiver Teilnehmer: Kongressteilnahmen: Novartis, Pharma Mar; Nichtfinanzielle Interessen: Oberarzt, Universitätsklinikum Würzburg, Medizinische Klinik und Poliklinik II; Mitgliedschaften: Deutsche Gesellschaft für Innere Medizin (DGIM), Deutsche Gesellschaft für Hämatologie und Medizinische Onkologie (DGHO), Arbeitsgemeinschaft der in Bayern tätigen Notärzte (AGBN).

S. Kraus: Finanzielle Interessen: Referentenhonorar oder Kostenerstattung als passiver Teilnehmer: Kongressteilnahmen: Celgene, Jazz, Medac, Novartis; Nichtfinanzielle Interessen: Oberärztin, Universitätsklinikum Würzburg, Medizinische Klinik und Poliklinik II; Mitgliedschaften: Deutsche Gesellschaft für Innere Medizin (DGIM), Deutsche Gesellschaft für Hämatologie und Medizinische Onkologie (DGHO), Deutsche Arbeitsgemeinschaft für Knochenmark- und Blutstammzelltransplantation (DAG-KBT), European Society for Blood and Marrow Transplantation (EBMT).

\section{Literatur}

[1] You H, Xu D, Zhao J et al. JAK Inhibitors: Prospects in Connective Tissue Diseases. Clin Rev Allergy Immunol 2020. https://doi. org/10.1007/s12016-020-08786-6 (Online ahead of print)

[2] Rampal R, Al-Shahrour F, Abdel-Wahab O et al. Integrated genomic analysis illustrates the central role of JAK-STAT pathway activation in myeloproliferative neoplasm pathogenesis. Blood 2014; 123: e123-133
[3] Zeiser R, Burchert A, Lengerke C et al. Ruxolitinib in corticosteroidrefractory graft-versus-host disease after allogeneic stem cell transplantation: a multicenter survey. Leukemia 2015; 29: 2062-2068

[4] Witte T. JAK-Inhibitoren in der Rheumatologie. Dtsch Med Wochenschr 2019; 144: 748-752

[5] Ajayi S, Becker H, Reinhardt $\mathrm{H}$ et al. Ruxolitinib. Recent Results Cancer Res 2018; 212: 119-132

[6] Tefferi A.. Myeloproliferative neoplasms: A decade of discoveries and treatment advances. Am J Hematol 2016; 91: 50-58

[7] Arber DA, Orazi A, Hasserjian R et al. The 2016 revision to the World Health Organization classification of myeloid neoplasms and acute leukemia. Blood 2016; 127: 2391-2405

[8] Tremblay D, Schwartz M, Bakst R et al. Modern management of splenomegaly in patients with myelofibrosis. Ann Hematol 2020; 99: 1441-1451

[9] Kröger NM, Deeg JH, Olavarria E et al. Indication and management of allogeneic stem cell transplantation in primary myelofibrosis: a consensus process by an EBMT/ELN international working group. Leukemia 2015; 29: 2126-2133

[10] Iurlo A, Cattaneo D, Gianelli U. Blast Transformation in Myeloproliferative Neoplasms: Risk Factors, Biological Findings, and Targeted Therapeutic Options. Int J Mol Sci 2019; 20

[11] Tallarico M, Odenike O. Secondary acute myeloid leukemias arising from Philadelphia chromosome negative myeloproliferative neoplasms: pathogenesis, risk factors, and therapeutic strategies. Curr Hematol Malig Rep 2015; 10: 112-117

[12] Kennedy JA, Atenafu EG, Messner HA et al. Treatment outcomes following leukemic transformation in Philadelphia-negative myeloproliferative neoplasms. Blood 2013; 121: 2725-2733

[13] Morris R, Kershaw NJ, Babon J]. The molecular details of cytokine signaling via the JAK/STAT pathway. Protein Sci 2018; 27: 1984-2009

[14] Tiong IS, Casolari DA, Moore S et al. Apparent 'JAK2-negative' polycythaemia vera due to compound mutations in exon $14 . \mathrm{Br}$ J Haematol 2017; 178: 333-336

[15] Greenfield G, McPherson S, Mills K et al. The ruxolitinib effect: understanding how molecular pathogenesis and epigenetic dysregulation impact therapeutic efficacy in myeloproliferative neoplasms. J Transl Med 2018; 16: 360

[16] Vainchenker W, Kralovics R. Genetic basis and molecular pathophysiology of classical myeloproliferative neoplasms. Blood 2017; 129: 667-679

[17] Nangalia J, Grinfeld J, Green AR. Pathogenesis of Myeloproliferative Disorders. Annu Rev Pathol 2016; 11: 101-126

[18] Tefferi A, Guglielmelli P, Larson DR et al. Long-term survival and blast transformation in molecularly annotated essential thrombocythemia, polycythemia vera, and myelofibrosis. Blood 2014; 124: 2507-2513. quiz 2615

[19] Milosevic Feenstra JD, Nivarthi H, Gisslinger $\mathrm{H}$ et al. Whole-exome sequencing identifies novel MPL and JAK2 mutations in triple-negative myeloproliferative neoplasms. Blood 2016; 127: 325-332

[20] Rumi E, Barate C, Benevolo G et al. Myeloproliferative and lymphoproliferative disorders: State of the art. Hematol Oncol 2020; 38: 121-128

[21] Harrison CN, Schaap N, Mesa RA. Management of myelofibrosis after ruxolitinib failure. Ann Hematol 2020; 99: 1177-1191

[22] Cervantes F, Dupriez B, Pereira A et al. New prognostic scoring system for primary myelofibrosis based on a study of the International Working Group for Myelofibrosis Research and Treatment. Blood 2009; 113: 2895-2901

[23] Passamonti F, Cervantes F, Vannucchi AM et al. A dynamic prognostic model to predict survival in primary myelofibrosis: a study by the IWG-MRT (International Working Group for Myeloproliferative Neoplasms Research and Treatment). Blood 2010; 115: 1703-1708 
[24] Gangat N, Caramazza D, Vaidya R et al. DIPSS plus: a refined Dynamic International Prognostic Scoring System for primary myelofibrosis that incorporates prognostic information from karyotype, platelet count, and transfusion status. J Clin Oncol 2011; 29: 392-397

[25] Guglielmelli P, Lasho TL, Rotunno G et al. MIPSS70: Mutation-Enhanced International Prognostic Score System for Transplantation-Age Patients With Primary Myelofibrosis. J Clin Oncol 2018; 36: 310-318

[26] Tefferi A, Guglielmelli P, Lasho TL et al. MIPSS70 + Version 2.0: Mutation and Karyotype-Enhanced International Prognostic Scoring System for Primary Myelofibrosis. J Clin Oncol 2018; 36: 1769-1770

[27] Tefferi A, Guglielmelli P, Nicolosi M et al. GIPSS: genetically inspired prognostic scoring system for primary myelofibrosis. Leukemia 2018; 32: 1631-1642

[28] Barbui T, Tefferi A, Vannucchi AM et al. Philadelphia chromosomenegative classical myeloproliferative neoplasms: revised management recommendations from European LeukemiaNet. Leukemia 2018; 32: 1057-1069

[29] Vannucchi AM, Kiladjian J], Griesshammer M et al. Ruxolitinib versus standard therapy for the treatment of polycythemia vera. $\mathrm{N}$ Engl J Med 2015; 372: 426-435

[30] DGHO. Deutsche Gesellschaft für Hämatologie und Onkologie (DGHO). Leitlinienportal Onkopedia. Verfügbar unter https://www. onkopedia.com/de/onkopedia/guidelines Zugegriffen: 02.09.2020

[31] Tiribelli M, Palandri F, Sant'Antonio E et al. The role of allogeneic stem-cell transplant in myelofibrosis in the era of JAK inhibitors: a case-based review. Bone Marrow Transplant 2020; 55: 708-716

[32] McLornan DP, Yakoub-Agha I, Robin M et al. State-of-the-art review: allogeneic stem cell transplantation for myelofibrosis in 2019. Haematologica 2019; 104: 659-668

[33] Shanavas M, Popat U, Michaelis LC et al. Outcomes of Allogeneic Hematopoietic Cell Transplantation in Patients with Myelofibrosis with Prior Exposure to Janus Kinase 1/2 Inhibitors. Biol Blood Marrow Transplant 2016; 22: 432-440

[34] Verstovsek S, Mesa RA, Gotlib J et al. The clinical benefit of ruxolitinib across patient subgroups: analysis of a placebo-controlled, Phase III study in patients with myelofibrosis. Br J Haematol 2013; 161: 508-516

[35] Verstovsek S, Kantarjian H, Mesa RA et al. Safety and efficacy of INCB018424, a JAK1 and JAK2 inhibitor, in myelofibrosis. N Engl J Med 2010; 363: 1117-1127

[36] Verstovsek S, Mesa RA, Gotlib J et al. A double-blind, placebo-controlled trial of ruxolitinib for myelofibrosis. N Engl J Med 2012; 366 : 799-807

[37] Harrison C, Kiladjian J], Al-Ali HK et al. JAK inhibition with ruxolitinib versus best available therapy for myelofibrosis. N Engl J Med 2012; 366: 787-798

[38] Al-Ali HK, Griesshammer M, Foltz L et al. Primary analysis of JUMP, a phase 3b, expanded-access study evaluating the safety and efficacy of ruxolitinib in patients with myelofibrosis, including those with low platelet counts. Br J Haematol 2020; 189: 888-903

[39] Verstovsek S, Gotlib J, Mesa RA et al. Long-term survival in patients treated with ruxolitinib for myelofibrosis: COMFORT-I and -II pooled analyses. J Hematol Oncol 2017; 10: 156

[40] Quintás-Cardama A, Vaddi K, Liu P et al. Preclinical characterization of the selective JAK1/2 inhibitor INCB018424: therapeutic implications for the treatment of myeloproliferative neoplasms. Blood 2010; 115: 3109-3117

[41] Bose P, Verstovsek S. JAK Inhibition for the Treatment of Myelofibrosis: Limitations and Future Perspectives. Hemasphere 2020; 4: e424

[42] Rudolph J, Heine A, Quast T et al. The JAK inhibitor ruxolitinib impairs dendritic cell migration via off-target inhibition of ROCK. Leukemia 2016; 30: 2119-2123
[43] Pemmaraju N, Kantarjian H, Nastoupil L et al. Characteristics of patients with myeloproliferative neoplasms with lymphoma, with or without JAK inhibitor therapy. Blood 2019; 133: 2348-2351

[44] Porpaczy E, Tripolt S, Hoelbl-Kovacic A et al. Aggressive B-cell lymphomas in patients with myelofibrosis receiving JAK1/2 inhibitor therapy. Blood 2018; 132: 694-706

[45] Rumi E, Zibellini S. JAK inhibitors and risk of B-cell lymphomas. Blood 2019; 133: 2251-2253

[46] Tefferi A, Pardanani A. Serious adverse events during ruxolitinib treatment discontinuation in patients with myelofibrosis. Mayo Clin Proc 2011; 86: 1188-1191

[47] Coltro G, Mannelli F, Guglielmelli P et al. A life-threatening ruxolitinib discontinuation syndrome. Am J Hematol 2017; 92: 833-838

[48] Newberry KJ, Patel K, Masarova L et al. Clonal evolution and outcomes in myelofibrosis after ruxolitinib discontinuation. Blood 2017; 130: $1125-1131$

[49] Palandri F, Breccia M, Bonifacio M et al. Life after ruxolitinib: Reasons for discontinuation, impact of disease phase, and outcomes in 218 patients with myelofibrosis. Cancer 2020; 126: 1243-1252

[50] Pardanani A, Harrison C, Cortes JE et al. Safety and Efficacy of Fedratinib in Patients With Primary or Secondary Myelofibrosis: A Randomized Clinical Trial. JAMA Oncol 2015; 1: 643-651

[51] Harrison CN, Schaap N, Vannucchi AM et al. Janus kinase-2 inhibitor fedratinib in patients with myelofibrosis previously treated with ruxolitinib (JAKARTA-2): a single-arm, open-label, non-randomised, phase 2, multicentre study. Lancet Haematol 2017; 4: e317-e324

[52] Mullally A, Hood ], Harrison C et al. Fedratinib in myelofibrosis. Blood Adv 2020; 4: 1792-1800

[53] Talpaz M, Kiladjian J]. Fedratinib, a newly approved treatment for patients with myeloproliferative neoplasm-associated myelofibrosis. Leukemia 2020. https://doi.org/10.1038/s41375-020-0954-2 (Online ahead of print)

[54] Zhang Q, Zhang Y, Diamond S et al. The Janus kinase 2 inhibitor fedratinib inhibits thiamine uptake: a putative mechanism for the onset of Wernicke's encephalopathy. Drug Metab Dispos 2014; 42: 1656-1662

[55] lurlo A, Cattaneo D, Bucelli C. Management of Myelofibrosis: from Diagnosis to New Target Therapies. Curr Treat Options Oncol 2020; 21: 46

[56] Singer JW, Al-Fayoumi S, Ma $\mathrm{H}$ et al. Comprehensive kinase profile of pacritinib, a nonmyelosuppressive Janus kinase 2 inhibitor. J Exp Pharmacol 2016; 8: 11-19

[57] Mascarenhas ], Hoffman R, Talpaz M et al. Pacritinib vs Best Available Therapy, Including Ruxolitinib, in Patients With Myelofibrosis: A Randomized Clinical Trial. JAMA Oncol 2018; 4: 652-659

[58] Asshoff M, Petzer V, Warr MR et al. Momelotinib inhibits ACVR1/ALK2, decreases hepcidin production, and ameliorates anemia of chronic disease in rodents. Blood 2017; 129: 1823-1830

[59] Mesa RA, Kiladjian JJ, Catalano JV et al. SIMPLIFY-1: A Phase III Randomized Trial of Momelotinib Versus Ruxolitinib in Janus Kinase Inhibitor-Naive Patients With Myelofibrosis. J Clin Oncol 2017; 35: 3844-3850

[60] Harrison CN, Vannucchi AM, Platzbecker U et al. Momelotinib versus best available therapy in patients with myelofibrosis previously treated with ruxolitinib (SIMPLIFY 2): a randomised, open-label, phase 3 trial. Lancet Haematol 2018; 5: e73-e81

[61] Daver N, Cortes J, Newberry K et al. Ruxolitinib in combination with lenalidomide as therapy for patients with myelofibrosis. Haematologica 2015; 100: 1058-1063

[62] Martin PJ, Rizzo JD, Wingard JR et al. First- and second-line systemic treatment of acute graft-versus-host disease: recommendations of the American Society of Blood and Marrow Transplantation. Biol Blood Marrow Transplant 2012; 18: 1150-1163 
[63] Zeiser R, Blazar BR. Acute Graft-versus-Host Disease - Biologic Process, Prevention, and Therapy. N Engl J Med 2017; 377: 2167-2179

[64] Zhang L, Yu J, Wei W. Advance in Targeted Immunotherapy for Graft-Versus-Host Disease. Front Immunol 2018; 9: 1087

[65] Khoury H], Wang T, Hemmer MT et al. Improved survival after acute graft-versus-host disease diagnosis in the modern era. Haematologica 2017; 102: 958-966

[66] Spoerl S, Mathew NR, Bscheider M et al. Activity of therapeutic JAK 1/2 blockade in graft-versus-host disease. Blood 2014; 123: 3832-3842

[67] Jagasia M, Perales MA, Schroeder MA et al. Ruxolitinib for the treatment of steroid-refractory acute GVHD (REACH1): a multicenter, open-label phase 2 trial. Blood 2020; 135: 1739-1749

[68] Przepiorka D, Luo L, Subramaniam S et al. FDA Approval Summary: Ruxolitinib for Treatment of Steroid-Refractory Acute Graft-VersusHost Disease. Oncologist 2020; 25: e328-e334

[69] Zeiser R, von Bubnoff N, Butler J et al. Ruxolitinib for GlucocorticoidRefractory Acute Graft-versus-Host Disease. N Engl J Med 2020; 382: $1800-1810$
[70] Zeiser R, Socié G. The development of ruxolitinib for glucocorticoidrefractory acute graft-versus-host disease. Blood Adv 2020; 4: 3789-3794

[71] Modi B, Hernandez-Henderson M, Yang D et al. Ruxolitinib as Salvage Therapy for Chronic Graft-versus-Host Disease. Biol Blood Marrow Transplant 2019; 25: 265-269

[72] Jagasia M, Zeiser R, Arbushites M et al. Ruxolitinib for the treatment of patients with steroid-refractory GVHD: an introduction to the REACH trials. Immunotherapy 2018; 10: 391-402

[73] Khoury H], Langston AA, Kota VK et al. Ruxolitinib: a steroid sparing agent in chronic graft-versus-host disease. Bone Marrow Transplant 2018; 53: 826-831

[74] Abedin S, McKenna E, Chhabra S et al. Efficacy, Toxicity, and Infectious Complications in Ruxolitinib-Treated Patients with CorticosteroidRefractory Graft-versus-Host Disease after Hematopoietic Cell Transplantation. Biol Blood Marrow Transplant 2019; 25: 1689-1694

[75] Mannina D, Kröger N. Janus Kinase Inhibition for Graft-Versus-Host Disease: Current Status and Future Prospects. Drugs 2019; 79: 1499-1509 